\title{
Cutaneous Route of Administration
}

National Cancer Institute

\section{Source}

National Cancer Institute. Cutaneous Route of Administration. NCI Thesaurus. Code C38675.

Administration to the skin. 